\title{
EVALUACIÓN DE UNA NUEVA SISTEMATIZACIÓN DE ESTUDIOS PARA EL DIAGNÓSTICO DEL SÍNDROME DE DESTRUCCIÓN VERTEBRAL
}

\author{
AVALIAĈ̃O DE NOVA SISTEMATIZAÇÃO DE EXAMES PARA O DIAGNÓSTICO \\ DA SINDROME DE DESTRUIÇÃO VERTEBRAL

\section{EVALUATION OF A NEW SYSTEMATIZATION OF TESTS FOR THE DIAGNOSIS OF VERTEBRAL DESTRUCTION SYNDROME}

Armando Alpizar Aguirre', luis Miguel Rosales Olivares², Guadalupe Sánchez Bringas' ${ }^{1}$, Barón Zarate Kalfopulos', Josue Giovanni Escutia García ${ }^{3}$, Alejandro Reyes Sánchez ${ }^{4}$

\section{RESUMEN}

Objetivo: Explorar la efectividad de un conjunto de pruebas de diagnósticos, sistematizadas en hoja de protocolo, para la determinación del Síndrome de Destrucción Vertebral en pacientes de nuestro servicio. Métodos: Analizamos una propuesta de sistematización mediante un estudio piloto de 20 pacientes a quienes se realizaron 10 diferentes pruebas de laboratorio y gabinete, además del estudio histopatológico de biopsia percutánea. Se describieron las características demográficas y clínicas, con los resultados obtenidos mediante estadística descriptiva, media y desviación estándar para las variables cuantitativas y porcentajes para las cualitativas. Resultados: Edad promedio de 53,3 años $\pm 16,56$ años; 10 hombres y 10 mujeres. 30 vértebras afectadas en los 20 pacientes, 8 de ellos tuvieron afección de dos o más vértebras. El nivel más afectado fue el Lumbar en 18 casos, seguido por el Torácico en 12. El Mal de Pott se diagnosticó en 7 casos (35\%), Osteomielitis en 4 (20\%), Mieloma Múltiple en 2 (10\%), y Espondilodiscitis, Metástasis de Adenocarcinoma, Linfoma y Osteoporosis en un caso respectivamente (5\%). En 3 casos (15\%) no se obtuvo un diagnóstico específico. Conclusiones: Esta propuesta de sistematización fue útil para llegar al diagnóstico etiológico definitivo del SDV en 85\% de los casos, con la reducción en la cantidad de estudios realizados. Es necesario mayor número de pacientes para aumentar la evidencia de su utilidad.

Descriptores: Columna vertebral; Infección; Neoplasias de la columna vertebral; Tuberculosis de la columna vertebral; Osteoporosis.

\section{RESUMO}

Objetivo: Explorar a efetividade de um conjunto de exames diagnósticos sistematizados em folha de protocolo para determinação da síndrome de destruição vertebral em pacientes atendidos em nosso serviço. Métodos: Analisamos uma proposta de sistematização por meio de estudo piloto de 20 pacientes que foram submetidos a dez exames laboratoriais e de imagens distintos, além do estudo histopatológico de biópsia percutânea. Foram descritas as características demográficas e clínicas e os resultados foram obtidos por estatística descritiva, média e desvio padrão para as variáveis quantitativas e porcentagens para as qualitativas. Resultados: Média de idade de 53,3 \pm 16,56 anos; 10 homens e 10 mulheres. Trinta vértebras afetadas nos 20 pacientes; 8 deles tinham afecção em duas ou mais vértebras. O nível mais afetado foi o lombar em 18 casos, seguido pelo torácico em 12. A tuberculose da coluna vertebral (doença de Pott) foi diagnosticada em 7 casos (35\%), a osteomielite, em 4 (20\%), o mieloma múltiplo, em 2 (10\%) discite, metástase de adenocarcinoma, linfoma e osteoporose, cada um em um caso (5\%). Em 3 casos (15\%) não se obteve diagnóstico específico. Conclusões: Esta proposta de sistematização foi útil para chegar ao diagnóstico etiológico definitivo da SDV em 85\% dos casos, com redução da quantidade de exames realizados. É preciso estudar um número maior de pacientes para aumentar a evidência de sua utilidade.

Descritores: Coluna vertebral; Infecção; Neoplasias da coluna vertebral; Tuberculose da coluna vertebral; Osteoporose.

\begin{abstract}
Objective: To explore the effectiveness of a systematic set of diagnostic tests in a protocol sheet for determination of vertebral destruction syndrome in patients treated in our service. Methods: We analysed a proposal for systematization through a pilot study of 20 patients who underwent 10 different laboratory and imaging tests, as well as the histopathological study through percutaneous biopsy. We described the demographic and clinical characteristics and likewise the results obtained by means of descriptive statistics, mean and standard deviation for quantitative variables and percentage for qualitative variables. Results: Average age of $53.3 \pm 16.56$ years; 10 men and 10 women. Thirty vertebrae affected in 20 patients; 8 patients had disease in two or more vertebrae. The most affected level was the lumbar in 18 cases, followed by thoracic in 12. The spinal tuberculosis (Pott's disease) was detected in 7 cases (35\%), osteomyelitis in 4 (20\%), multiple myeloma, in 2 (10\%), and discitis, metastasis of adenocarcinoma, lymphoma and osteoporosis in one case (5\%). In 3 cases (15\%) there was no specific diagnosis. Conclusions: This proposal of systematization was useful to reach the final etiological diagnosis of the VDS in $85 \%$ of cases, with the reduction in the number of tests. It is necessary to study a larger number of patients to increase the evidence of its usefulness.
\end{abstract}

Keywords: Spine; Infection; Spinal neoplasms; Tuberculosis, Spinal; Osteoporosis.

1. Médico Adcrito al Servicio de Cirugía de Columna Vertebral, Instituto Nacional de Rehabilitación, Secretaria de Salud (INR, S. S.). México.

2. Jefatura de Servicio Cirugía de Columna Vertebral INR, Secretaria de Salud (INR, S. S.). México.

3. Residencia Ortopedia y Traumatología INR, Secretaria de Salud (INR, S. S.). México.

4. Jefe de División de Cirugía Especial, INR, Secretaria de Salud (INR, S. S.). México.

Trabajo realizado Secretaria de Salud (INR, S. S.). México.

Correspondencia: Alejandro A. Reyes Sánchez. Camino a Santa Teresa 1055-684, Col. Héroes de Padierna Del. Magdalena Contreras, México, D. F. CP 10700 - Mexico. E-mail: alereyes@ inr.gob.mx 


\section{INTRODUCCION}

Se define al síndrome de destrucción vertebral (SDV) como una patología de múltiple etiología, caracterizada por alteraciones en la estructura y ultraestructura de la columna vertebral; específicamente con deformidad y aumento de volumen circunvecino en uno o más cuerpos vertebrales. Se acompaña de dolor e incapacidad funcional por alteraciones mecánicas y neurológicas ${ }^{1}$.

La literatura reporta como causas del síndrome de destrucción vertebral a las siguientes: (1) Procesos Infecciosos: Bacterianos, Micobacterias, Hongos, y Parásitos; (2) Tumores Primarios: Benignos y Malignos; (3) Metástasis Tumorales: De otros tumores primarios; y (4) Alteraciones Metabólicas: Osteoporosis, Osteomalacia y Enfermedad de Paget entre otras².

El diagnóstico del conjunto de patologías que originan el SDV está en relación con datos clínicos, de gabinete, laboratorio, y hallazgos específicos para cada patología en estudios de gabinete ${ }^{3-15}$. Los estudios reportan la biopsia en general como el elemento más importante para el diagnóstico etiológico ${ }^{4,12}$. Sin embargo, Rosales et al. ${ }^{1}$ reportó la utilidad de la biopsia transpedicular percutánea guiada por fluoroscopía, para el diagnóstico de SDV en 20 pacientes. En donde la edad promedio de los pacientes al momento del estudio fue de 57 años, con predominio del sexo masculino (55\%); los segmentos estudiados fueron columna lumbar (70\%) y torácica (30\%); sin complicaciones derivadas del procedimiento en ningún paciente. A pesar que las cifras son coincidentes con gran mayoría de otras series, en este estudio se logró un diagnóstico histopatológico específico en sólo el 55\% de los pacientes, lo cual lo evidencia a la Biopsia como un elemento diagnóstico poco útil ${ }^{16}$.

Con ese antecedente, en 2007 Alpízar propone una sistematización con una serie de exámenes que incluyeron estudios de laboratorio, gabinete y biopsia percutánea, para llegar al diagnóstico etiológico del SDV². En su estudio se incluyeron 105 pacientes; en base a las distintas patologías, plantearon una sistematización y hoja de registro de los estudios en 3 bloques: infecciones, tumores, y enfermedades metabólicas.

Como conclusión general del estudio, se encontró que se difiere en algunos resultados de sensibilidad y especificidad de los estudios diagnósticos, respecto a la literatura mundial ${ }^{17}$. Así mismo, se propone una nueva sistematización simplificada a partir de los resultados obtenidos en dicho protocolo, utilizando los estudios con mayor sensibilidad y especificidad a la vez que se pretende disminuir los costos, sin afectar e incluso mejorar la efectividad del método diagnóstico.

En la literatura mundial hasta ahora, la biopsia abierta constituye el estándar de referencia para el diagnóstico con un 98\% de precisión ${ }^{12}$. Con técnica percutánea, se reporta con especificidades desde el 71 hasta el $100 \%{ }^{1}$, sin relevancia significativa en las variaciones de la técnica, relativas al diámetro de la trefina o al método de guía imagenológica de la toma ${ }^{12}$.

En la literatura anglosajona, la búsqueda de la etiología del SDV prescinde de una estandarización, y se aborda el estudio desde la sospecha diagnóstica ${ }^{7}$. Por tanto, la falta de una sistematización para el diagnóstico, deja el protocolo de estudio a criterio del médico tratante. Es en este país, que Alpízar et al. $^{2}$ propone un protocolo sistematizado, para llegar al diagnóstico etiológico en pacientes de primera vez que cursan con SDV.

Basado a su vez en los estudios de Rosales publicados en el 2006, sobre la utilidad en la biopsia percutánea para el diagnóstico etiológico ${ }^{1}$. Alpízar parte del resultado obtenido: sólo en el 55\% de los pacientes se obtuvo el diagnóstico histopatológico específico del SDV. Por esto, se decide la inclusión de 25 estudios de laboratorio y gabinete, incluida la biopsia, para llegar al diagnóstico. Entre los hallazgos publicados en 2007 se tuvieron algunos estudios como poco relevantes para efectos diagnósticos del SDV, teniendo con mayor sensibilidad y especificidad la Resonancia Magnética, la Tomografía Axial Computada, la Reacción en Cadena de la Polimerasa para TB, así como el Estudio Gammagráfico; motivo por el cual ellos proponen replantear esta sistematización?2.
Según Alpízar et al. ${ }^{2}$, la destrucción vertebral aún prescinde de un algoritmo diagnóstico efectivo, rápido, y con menor impacto económico para el paciente. Por lo anterior, se deben lograr todos éstos objetivos con el análisis de los resultados obtenidos de la sistematización, respecto a sensibilidades y especificidades de los diversos estudios; de tal manera, que sea reproducible en otros centros hospitalarios.

Contamos con el antecedente que Rosales ${ }^{1}$ encontró que la biopsia sólo tiene una efectividad del 55\% para el diagnóstico etiológico del SDV, lo que nos lleva a continuar la línea de investigación propuesta. Aunque previamente se analizó la sensibilidad y especificidad de los diversos estudios de manera independiente, el estudio de Alpízar et al. ${ }^{2}$ pone en evidencia sus índices diagnósticos para el estudio específico del SDV.

Para las infecciones, lo más sensible fue la Gammagrafía con un $60 \%$. A pesar de la baja sensibilidad de los laboratorios y la Resonancia Magnética (25 y 14.3\% respectivamente), la especificidad de éstas (100 y 98.1\%) fue similar a la de la Gammagrafía, del 94.3\%. El valor predictivo positivo mayor fue para el laboratorio con un $100 \%$, seguido de la Gammagrafía con un $66.6 \%$; las mayores exactitudes correspondieron al laboratorio y la Resonancia Magnética con un 93 y $91.5 \%$.

En infecciones granulomatosas, los laboratorios tuvieron una sensibilidad del 69.2\%, la Resonancia Magnética y la Gammagrafía tuvieron una baja sensibilidad (18.2 y 38.5\% respectivamente); sin embargo, todas ellas resultaron con una especificidad y valor predictivo positivo del 100\%; su exactitud fue de 90.6 , 88.1 y $87 \%$ respectivamente.

En lo respectivo al diagnóstico del mieloma múltiple, los laboratorios tuvieron una sensibilidad de 44.4\%, Resonancia Magnética de $12.5 \%$ y Gammagrafía de $42.9 \%$; las especificidades fueron del $100,98.1$ y $96.4 \%$ respectivamente. Los laboratorios alcanzaron un valor predictivo positivo del 100\%; y la exactitud de las pruebas fue de $88.3 \%$ para los mismos, contra $90.4 \%$ de Resonancia Magnética y Gammagrafía.

Para los tumores primarios, el más sensible fue la Resonancia Magnética con un 54.5\%; los laboratorios no fueron sensibles. La mayor especificidad, sin embargo, corresponde con un 100\% a éstos últimos, seguido de la Gammagrafía con un 94.3\%. El valor predictivo positivo mayor fue de $66.6 \%$ de la Resonancia Magnética; la mayor exactitud correspondió a los laboratorios con 88.3\%, seguido de la Resonancia Magnética con un 86.8\%.

En los tumores secundarios, la Gammagrafía fue el estudio más sensible con un75\%. Sin embargo, los laboratorios fueron los más específicos con un 100\%; seguido de la Resonancia Magnética con un $98.2 \%$ y la Gammagrafía con un 96.6\%. El valor predictivo positivo mayor correspondió a la Tomografía Axial Computada con un 100\%. La mayor exactitud fue para la Tomografía Axial Computada con un 96.4\%; sin embargo, fue en este rubro donde la Resonancia Magnética y la Gammagrafía obtuvieron valores muy cercanos, con un 95 y $95.2 \%$ respectivamente.

En base a los hallazgos de Alpízar et al. ${ }^{2}$, se toman en cuenta los 10 estudios de su sistematización previa, de los cuales se encontró mayor utilidad para el diagnóstico del SDV², que son; Biometría Hemática; Química Sanguínea; Tiempos de Coagulación; Examen General de Orina; Proteína de Bence-Jones; Serología para VIH; Reacción en Cadena de la Polimerasa para Tuberculosis (PCR); Rayos X simples y Lateral de Cráneo; Resonancia Magnética; Gammagrafía; Biopsia.

Con el objetivo general de explorar la efectividad de este conjunto de pruebas diagnósticas sistematizadas en hoja de protocolo, para la determinación del Síndrome de Destrucción Vertebral en pacientes de nuestro servicio. Inicialmente realizamos un estudio piloto con una muestra de 20 pacientes, en quienes se aplicó la sistematización y se valoró su viabilidad para el diagnóstico etiológico del SDV.

Através de los objetivos secundarios de describir las variables demográficas; describir los sitios de mayor frecuencia de presentación del SDV; y cuantificar los resultados para las diferentes pruebas propuestas en la hoja de protocolo. 
Asumimos como hipótesis que el conjunto de pruebas diagnósticas sistematizadas en la hoja de protocolo representa un método completo y suficiente para determinar la etiología del Síndrome de Destrucción Vertebral, en nuestro servicio.

\section{MATERIAL Y MÉTODOS}

Se realizó un estudio piloto prospectivo, transversal, en 20 pacientes captados en nuestro servicio, que acudieron a los servicios de Consulta Externa o Urgencias en el período comprendido entre Agosto de 2006 a Diciembre de 2008; con SDV sin diagnóstico etiológico. Todos los pacientes se Hospitalización para la realización de los siguientes estudios: Biometría Hemática con Diferencial, Química Sanguínea, Tiempos de Protrombina y Parcial de Tromboplastina, Examen General de Orina, Proteína de Bence-Jones, Virus de Inmunodeficiencia Humana, Reacción en Cadena de Polimerasa para Tuberculosis, Rayos X simples, y AP y Lateral de Cráneo, Resonancia Magnética, Gammagrafía y Biopsia.

Los pacientes permanecieron hospitalizados hasta realizar la totalidad de los estudios del protocolo. Dependiendo de su estado clínico, algunos pacientes permanecieron hospitalizados para su tratamiento quirúrgico definitivo, mientras que otros se egresaron en espera de su resultado histopatologico, y programación posterior de su tratamiento quirúrgico y médico especifico.

La recolección de resultados se hizo a través del llenado del formato correspondiente del protocolo, el cual incluye las 10 pruebas propuestas. (Figura 1). Y se anexaron al expediente. Para los estudios de laboratorio, se manejaron como normales las cifras dentro de los rangos mostrados en la Cuadro 1. Para los estudios específicos, se manejaron en la base de datos como Positivos ó Negativos para el diagnóstico etiológico del SDV.

Se realizó la estadística descriptiva de los datos, la media y desviación estándar para los datos cuantitativos y porcentajes para los datos cualitativos. Se utilizaron tablas y gráficas de salida. La base de datos se recabó en hoja de cálculo del programa Excel de Microsoft. El análisis se llevó a cabo con el paquete estadístico SPSS-15.

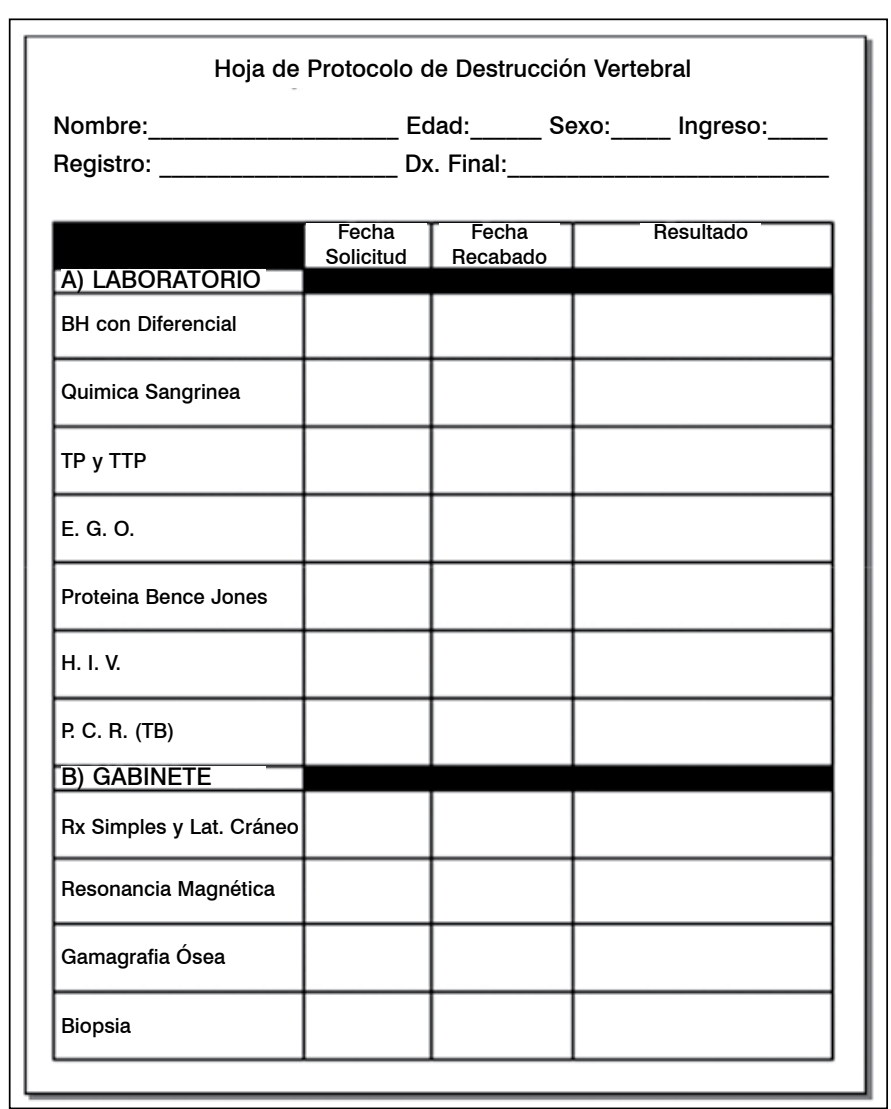

Figura 1. Formato de llenado del Protocolo de Destrucción Vertebral.
Cuadro 1. Rangos establecidos para normalidad en pruebas de laboratorio.

\begin{tabular}{|c|c|c|c|}
\hline Parametro & Rango minimo & Rango maximo & Unidades \\
\hline Hemoglobina & 11.0 & 16.5 & $\mathrm{~g} / \mathrm{dl}$ \\
\hline Hematocrito & 35.0 & 50.0 & $\%$ \\
\hline Leucocitos & 3.5 & 10.0 & $\times 10^{3} / \mathrm{mm}^{3}$ \\
\hline Glucosa & 70 & 105 & $\mathrm{mg} / \mathrm{dl}$ \\
\hline Urea & 15 & 39 & $\mathrm{mg} / \mathrm{dl}$ \\
\hline Creatinina & 0.5 & 1.2 & $\mathrm{mg} / \mathrm{dl}$ \\
\hline TP & 12.0 & 14.5 & Segundos \\
\hline TTP & 25 & 45 & Segundos \\
\hline
\end{tabular}

TP tiempo de Protrombina, TTP Tiempo de TromboPlastina.

\section{RESULTADOS}

En nuestro servicio se han atendido 195 pacientes con SDV desde 1996 hasta el cierre del 2008. Del periodo de agosto de 2006 a diciembre de 2008 se atendieron 82 pacientes con SDV, de los cuales se seleccionaron 20 pacientes con la sistematización de estudios completa, y recabada en la hoja de protocolo y expediente clínico.

La edad promedio de los 20 casos fue de 53.3 años \pm 16.56 años (rango 22 a 79 años); 10 del sexo masculino y 10 del femenino. La mayoría (45\%) tenían de 60 años en adelante (Cuadro 2). De los 20 pacientes se estudiaron 30 vértebras afectadas, encontrando que 8 pacientes tuvieron afección a dos o más vértebras, y obteniendo que el nivel más afectado fué el Lumbar con 18 eventos en total; seguido por el Torácico con 12 eventos en total (Cuadro 3).

De los 20 casos, 7 se diagnosticaron como Mal de Pott, 4 como Osteomielitis, 2 como Mieloma Múltiple, 1 como Espondilodiscitis, uno como Linfoma, uno como metástasis de Adenocarcinoma, uno como Osteoporosis, y en tres no se pudo obtener un diagnóstico específico (Figura 2).

Cuadro 2. Distribución por grupo etáreo y sexo.

\begin{tabular}{|c|c|c|}
\hline Edad (años) & Mujeres & Hombres \\
\hline $20-29$ & 2 & 0 \\
\hline $30-39$ & 0 & 3 \\
\hline $40-49$ & 1 & 1 \\
\hline $50-59$ & 1 & 3 \\
\hline Mayores de 60 & 6 & 3 \\
\hline
\end{tabular}

Cuadro 3. Casos registrados por región de la columna vertebral.

\begin{tabular}{|c|c|c|c|c|c|}
\hline No. Niveles & Nivel toracico & Nivel lumbar & Frecuencia & Porcentaje & Porcentaje acumulado \\
\hline 1 & 5 & 7 & 12 & 60 & 60 \\
\hline 2 & 3 & 4 & 7 & 35 & 95 \\
\hline Más de 2 & 0 & 1 & 1 & 5 & 100 \\
\hline Total & 8 & 12 & 20 & 100 & \\
\hline
\end{tabular}

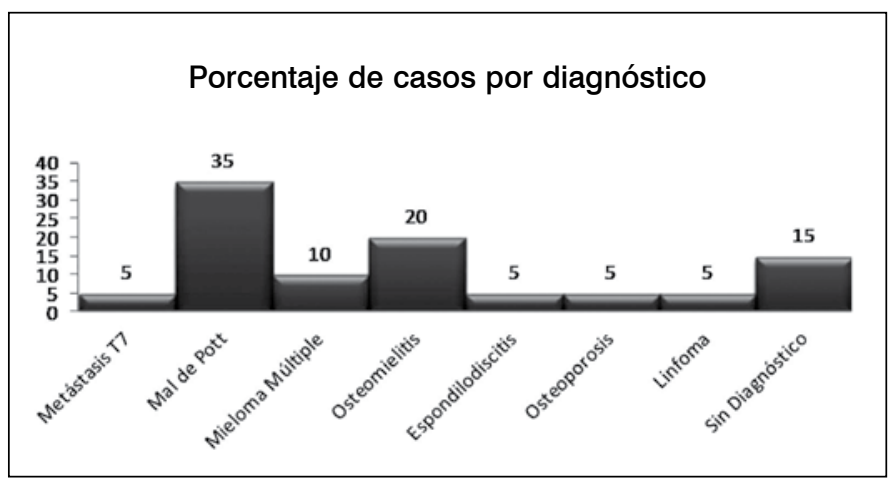

Figura 2. Porcentaje de casos por diagnóstico. 
En lo respectivo a los resultados de estudios, se describe como sigue: Para Mal de Pott, hubo variaciones en las pruebas realizadas para su diagnóstico específico:

- En uno de los casos hubo disminución en el Hematocrito;

- Solamente en uno hubo aumento de Leucocitos;

- Tres presentaron alteracion en la Glucemia;

- De los Azoados, en tres de ellos aumento la Uricemia sin aumento de la Creatinina en ninguno de los pacientes;

- Tres casos presentaron datos de infección en vías urinarias en el EGO;

- De las pruebas específicas, la RCP se mostró negativa en 3 casos a pesar de ser una prueba altamente específica; sin embargo el diagnóstico de ellos se obtuvo por Gammagrafía, biopsia, y en uno además con RM positivas para el diagnóstico. Cinco casos de los siete fueron positivos para Gammagrafía, y cinco de los siete fueron positivos para biopsia.

\section{Para Osteomielitis hubo cuatro casos; de estos:}

- En uno se presentó disminución de Hemoglobina y Hematocrito;

- En otros dos se presentó Leucocitosis, quienes al mismo tiempo presentaron; Hiperglucemia, Hiperuricemia, y uno más con discreto aumento de la Creatinina;

- Otros dos presentaron alargamiento del Tiempo de Protrombina;

- Tres de los casos con datos de infección en el Examen General de Orina;

- De las pruebas específicas, sólo uno dio positivo para la RM, y otros dos en la Gammagrafía. Ninguno dio positivo en el estudio de biopsia.

\section{El caso de Espondilodiscitis mostró lo siguiente:}

- Elevación de la Urea;

- Disminución del Tiempo de Protrombina;

- La RM fue el único estudio que dio positivo para el diagnóstico específico; la Gammagrafía y la biopsia dieron negativos para el diagnóstico;

- No hubo alteraciones en el resto de los estudios.

\section{Hubo dos casos de Mieloma Múltiple:}

- Uno de los casos presentó disminución en Hemoglobina y Hematocrito:

- Uno presentó Hiperuricemia;

- Uno con alargamiento de Tiempo de Protrombin;

- El EGO mostró un caso con datos de infección y uno con cristaluria;

- Radiológicamente ambos casos dieron positivos con imágenes líticas en sacabocado en el cráneo;

- De las pruebas de Gammagrafía y biopsia, ambos casos dieron resultado positivo para el diagnóstico; además que uno de ellos dio imágenes positivas características en la RM. Sin embargo, la prueba de búsqueda de PBJ dio negativo en ambos casos.

El caso de Linfoma mostró Leucocitosis y discreta elevación de la Urea, y EGO con datos de infección. El diagnóstico definitivo se hizo por Gammagrafía y biopsia.

\section{El caso de Metástasis de Adenocarcinoma arrojó los siguientes resultados:}

- Leucocitosis;

- Hiperuricemia con Creatinina normal;

- Alargamiento del Tiempo de Protrombina;

- EGO con datos de infección:

- Este caso además presentó RCP positiva para Tuberculosis, lo cual dió otra alternativa diagnóstica; sin embargo por imágenes características positivas en RM, así como el estudio histopatológico, dieron como resultado el diagnóstico de metástasis de Adenocarcinoma.

El caso de Osteoporosis no mostró alteraciones en ninguno de los estudios además del colapso del cuerpo vertebral, que pudo evidenciarse con estudios de imagen.

\section{Los tres casos sin diagnóstico específico dieron los siguientes resultados:}

- Uno mostró discreta disminución del Hematocrito;
- Dos de ellos resultaron con Hiperuricemia sin alteraciones en la Creatinina;

- Uno de ellos resultó con disminución de los Tiempos de Protrombina y Parcial de Tromboplastina; otro de ellos, con datos de infección en el EGO;

Uno de ellos sólo mostró tejido con esclerosis en la biopsia; los otros dos sólo con cambios inespecíficos. Los estudios de imagen sin cambios que orientasen al diagnóstico;

En lo respectivo a las pruebas, el Examen General de Orina salió positivo para infección en 10 casos, normal en 8 casos, cristaluria en un caso y hematuria en un caso; sin embargo la prueba es inespecífica para cualquiera de los diagnósticos mencionados. No se encontraron pacientes con prueba positiva para PBJ ni serología para $\mathrm{VIH}$. La RCP fue positiva en 5 casos; la radiología para Mieloma Múltiple positiva en 2 casos, la RM con 6 casos positivos, Gammagrafía y biopsia con 10 casos positivos para diagnóstico respectivamente (Figura 3 ).

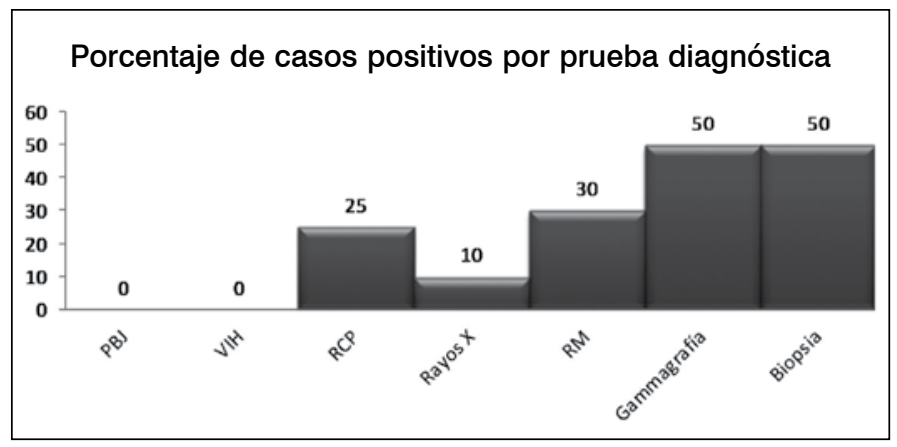

Figura 3. Porcentaje de casos positivos por prueba diagnóstica.

\section{DISCUSIÓN}

En el presente estudio, se evaluó la efectividad de la hoja de protocolo para obtener el diagnóstico etiológico del Síndrome de Destrucción Vertebral. Con respecto al género, los pacientes estudiados fueron 10 femeninos, y 10 masculinos. Por edad, el mayor porcentaje correspondió al grupo etáreo de personas mayores de 60 años con un $45 \%$ de los casos y de ellos, mayor predominio del sexo femenino; el segmento más afectado fue el Lumbar. Los resultados obtenidos respecto al diagnóstico confirmaron la mayor frecuencia del Mal de Pott con 7 casos (35\%), lo que coincide con el estudio de Alpízar y colaboradores ${ }^{2}$. Siguieron en frecuencia, la Osteomielitis con 4 casos (20\%), y el Mieloma Múltiple con 2 casos (10\%), y se presentaron Espondilodiscitis, Metástasis de Adenocarcinoma, Linfoma y Osteoporosis con un caso (5\%), respectivamente. En 3 de los pacientes (15\%) no se pudo obtener un diagnóstico específico a partir de la hoja de protocolo.

En base a los resultados, y según lo ya estudiado por Alpízar et al. $^{2}$, podemos diferenciar los estudios realizados entre Pruebas Generales Auxiliares y Pruebas Específicas. Dentro de las primeras, se realizaron: Biometría Hemática, Química Sanguínea, Tiempos de Protrombina y Parcial de Tromboplastina, Examen General de Orina, y serología para $\mathrm{VIH}$, cuya utilidad fue limitada por su insensibilidad o inespecificidad para obtener un diagnóstico etiológico definitivo? Es conveniente hacer notar que la prueba serológica positiva para $\mathrm{VIH}$, ayuda a valorar la posibilidad de que exista inmunocompromiso del paciente, lo cual es un factor que predispone a algunas infecciones oportunistas descritas como etiología del SDV6.7.

Dentro de las pruebas específicas, la prueba de búsqueda de PBJ resultó negativa en ambos pacientes con Mieloma Múltiple; una posible explicación se encuentra en los trabajos de Beetham ${ }^{17}$ quien demuestra que en $35 \%$ de los laboratorios no se puede detectar ésta proteína a una concentración aproximada de 60mg/L, además que su detección depende de factores tanto biológicos como del método de detección. El mismo autor reporta que la proporción excretada de PBJ para su detección depende de su metabolismo 
y reabsorción en base a la función renal del paciente; así como la relación de existencia de otras proteínas o cadenas ligeras de inmunoglobulinas mono o policlonales. Pero un estudio más reciente de $\mathrm{Lin}^{18}$, reporta que la PBJ puede encontrarse hasta en un $80 \%$ de los pacientes, y en los sintomáticos puede ser hasta de $1 \mathrm{~g} / \mathrm{d}$ en orina.

La RCP resultó positiva para tuberculosis en un 25\% de los casos, lo que correspondió a 4 de los 7 que se presentaron con Mal de Pott; el caso positivo restante fue del paciente con Metástasis de Adenocarcinoma, lo cual puede explicarse teniendo en cuenta una posible infección por Mycobacterium tuberculosis en un foco no vertebral, dada la especificidad de la prueba ${ }^{5}$.

En ambos casos de Mieloma Múltiple, se mantuvo la constante de hallazgos característicos de imágenes en "sacabocado" en las proyecciones radiológicas del cráneo; sin embargo, Togawa et al. ${ }^{16}$ reportaron que ésta enfermedad puede manifestarse inicialmente en la columna vertebral dando lugar a fracturas patológicas, sin que se detecten otros hallazgos hasta realizar el estudio histopatológico de biopsia vertebral. Para hacer el diagnóstico diferencial contra Osteoporosis u Osteomalacia, ellos sugieren la realización de la biopsia percutánea en el mismo evento quirúrgico en que se realiza la vertebroplastía.

La RM fue positiva en un 30\% del total de casos, y la Gammagrafía en 50\%; esta diferencia puede explicarse debido a que el tamaño de muestra, aunque fue adecuado para el tipo de estudio que se realizó, fue limitado respecto a las grandes series utilizadas para determinar sensibilidad y especificidad de los mismos además de su utilidad relativa a cada patología $a^{2,8,15}$. Se mantiene a la RM como la prueba de elección para detección de tumores ${ }^{4,15}$, comprobando su eficacia y especificidad en este estudio para detectar una metástasis tumoral de Adenocarcinoma y uno de los casos de Mieloma Múltiple. La Gammagrafía demostró alta eficacia para el diagnóstico de Mal de Pott en 5 de los 7 casos (71.42\%), en 2 de 4 de Osteomielitis (50\%), y en los 2 de Mieloma Múltiple (100\%); sin embargo, para ésta última patología no se describe como estudio de elección dada la limitada reacción ósea a la lisis tumoral ${ }^{13}$. La alta sensibilidad de la gammagrafía es de mayor utilidad para procesos infecciosos; sin embargo, la mayor sensibilidad es atribuida a la $\mathrm{RM}^{8}$, incluso para delimitar el sitio exacto de infección, como se demuestra para el caso de espondilodiscitis de este estudio.

En la literatura reciente, Nourbakhsh et al. ${ }^{12}$ reporta la persistencia de la Biopsia Abierta, como el estándar de referencia para el diagnóstico etiológico en los casos de destrucción vertebral ${ }^{12}$, incluso sugiere realizarla cuando no es posible obtener un diagnóstico definitivo por técnicas cerradas ${ }^{4}$. Con técnica percutánea, se reporta con especificidades desde el 71 hasta el $100 \%{ }^{1}$, sin relevancia significativa en las variaciones de la técnica, relativas al diámetro de la trefina o al método de guía imagenológica de la toma $^{12}$, y se dispone de las modalidades descritas por trefina ó por aspiración con aguja, guiadas con TC o fluoroscopía. En este estudio se llevó a cabo la técnica percutánea guiada con fluoroscopía. Se ha reportado como desventaja de esta técnica, el que a pesar de obtener imágenes en tiempo real, no es posible corroborar de manera exacta la obtención de material del sitio preciso de la lesión, situación que podría explicar que en algunos casos, el diagnóstico histopatológico no sea contundente $e^{9,10,11}$. Sin embargo, obteniendo un $50 \%$ de pruebas positivas en este estudio, se mantiene una cifra aproximada a los resultados de la biopsia percutánea reportados previamente por Rosales y colaboradores ${ }^{1}$.

En este estudio piloto se obtuvieron resultados similares a los reportes previos hechos en este país por Rosales et al. ${ }^{1}$ y Alpízar et al.2; cabe mencionar que el tamaño de muestra, justificado por el carácter exploratorio de este trabajo, fue una limitante para realizar el análisis de sensibilidad y especificidad. Sin embargo, esta nueva propuesta de sistematización de estudios, por un lado, ha sido útil para llegar al diagnóstico etiológico definitivo del SDV en la mayoría de los casos y por el otro, sustenta las bases para realizar la validación de la hoja de protocolo como herramienta diagnóstica del Síndrome de Destrucción Vertebral en el Instituto Nacional de Rehabilitación, y en una siguiente etapa de validación multicéntrica.

\section{CONCLUSIONES}

En este estudio piloto se realizó la descripción de una nueva sistematización de estudios, con la cual se pretende optimizar los recursos hospitalarios y, al mismo tiempo, hacer más efectivo el diagnóstico del SDV. Se logró reducir la cantidad de estudios a 10 pruebas diagnósticas además de la biopsia percutánea. Los resultados obtenidos de las pruebas realizadas a los 20 pacientes, fueron similares a aquellos de la serie de Alpízar y colaboradores, lo que derivó en el diseño de este nuevo protocolo; así mismo, fueron similares a los resultados del diagnóstico histopatológico por biopsia percutánea, previamente estudiado por Rosales y colaboradores. Sin embargo, en otras pruebas específicas como la Resonancia Magnética y la Gammagrafía observamos diferencias en cuanto a determinación del diagnóstico, por lo que en base a los resultados de este estudio se considera que es posible abordar una siguiente etapa de investigación con un nivel de evidencia mayor, y con el objetivo de determinar la tendencia de los resultados de las pruebas en relación a otras patologías que quedaron por determinarse en el presente análisis. De esa manera, podremos validar la hoja de protocolo para el amplio espectro de patologías causantes del SDV.

\section{REFERENCIAS}

1. Rosales-Olivares LM, Valle-Cerna I, Alpizar-Aguirre A, Miramontes-Martínez V, Arenas-Sordo Mde L, Reyes-Sánchez A. Percutaneous biopsy evaluation in the diagnosis of thoracic and lumbar vertebral destruction syndrome. Cir Cir. 2007;75(6):459-63.

2. Alpízar-Aguirre A, Mireles-Cano JN, Rosales-Olivares M, Miramontes-Martínez V, Reyes-Sánchez A. Clinical and radiological follow-up of Nubac disc prosthesis. Preliminary report. Cir Cir. 2008;76(4):317-21.

3. Lew DP, Waldvogel FA. Osteomyelitis. N Engl J Med. 1997;336(14):999-1007.

4. McLain RF, Weinstein JN, Spine Tumors In: Herkowitz HN, Garfin S R, Eismont F J, Bell G R, Balderston R A. Rothman-Simeone The Spine. 5th ed. Philadelphia: Editorial Saunders Elsevier; 2006. p.1233-354.

5. Jaramillo-de la Torre JJ, Bohinski RJ, Kuntz C 4th. Vertebral osteomyelitis. Neurosurg Clin N Am. 2006:17(3):339-51.

6. Chuo CY, FuYC, LuYM, Chen JC, Shen WJ, Yang CH, et al. Spinal infection in intravenous drug abusers. J Spinal Disord Tech. 2007;20(4):324-8.

7. Kim CW, Perry A, Currier B, Yaszemski M, Garfin SR. Fungal infections of the spine. Clin Orthop Relat Res. 2006;(444):92-9.

8. Pineda C, Vargas A, Rodríguez AV. Imaging of osteomyelitis: current concepts. Infect Dis Clin North Am. 2006;20(4):789-825.

9. Hadjipavlou AG, Kontakis GM, Gaitanis JN, Katonis PG, Lander P, Crow WN. Effectiveness and pitfalls of percutaneous transpedicle biopsy of the spine. Clin Orthop Relat Res. 2003;(411):54-60.
10. Pierot $L$, Boulin A. Percutaneous biopsy of the thoracic and lumbar spine: transpedicular approach under fluoroscopic guidance. AJNR Am J Neuroradiol. 1999;20(1):23-5.

11. Krause ND, Haddad ZK, Winalski CS, Ready JE, Nawfel RD, Carrino JA. Musculoskeletal biopsies using computed tomography fluoroscopy. J Comput Assist Tomogr. 2008;32(3):458-62

12. Nourbakhsh A, Grady JJ, Garges KJ. Percutaneous spine biopsy: a meta-analysis. J Bone Joint Surg Am. 2008;90(8):1722-5.

13. Simmons ED, Zheng Y. Vertebral tumors: surgical versus nonsurgical treatment. Clin Orthop Relat Res. 2006;(443):233-47.

14. Khan SN, Donthineni R. Surgical management of metastatic spine tumors. Orthop Clin North Am. 2006;37(1):99-104.

15. Bloomer CW, Ackerman A, Bhatia RG. Imaging for spine tumors and new applications. Top Magn Reson Imaging. 2006;17(2):69-87.

16. Togawa D, Lieberman IH, Bauer TW, Reinhardt MK, Kayanja MM. Histological evaluation of biopsies obtained from vertebral compression fractures: unsuspected myeloma and osteomalacia. Spine. 2005;30(7):781-6.

17. Beetham R. Detection of Bence-Jones protein in practice. Ann Clin Biochem. 2000;37:563-70

18. Lin P. Plasma cell myeloma. Hematol Oncol Clin North Am. 2009;23(4):709-27. 